\title{
Modelo de ordenamiento territorial para el desarrollo ambiental sostenible de la región San Martín
}

\author{
Pedro Cunya Flores \\ pedrocunya@hotmail.com \\ ORCID: 0000-0003-1923-4395 \\ Escuela de posgrado \\ Universidad César Vallejo \\ Hipólito Percy Barbarán Mozo \\ pbarmozito@hotmail.com \\ ORCID: 0000-0002-9316-202X \\ Escuela de Posgrado \\ Universidad César Vallejo \\ Carlos E. Arévalo Arévalo \\ carlos.arevalo@unas.edu.pe \\ ORCID: 0000-0002-6626-7758
}

Helbert Vásquez Angulo

helvasan@hotmail.com

ORCID: 0000-0003-1556-2615

Escuela de posgrado

Universidad César Vallejo

Percy Lozano Gómez

pelogo83@gmail.com

ORCID: 000-001-6951-4163

Escuela de posgrado

Universidad César Vallejo

\section{RESUMEN}

El presente estudio tuvo como objetivo, determinar el estado situacional del desarrollo ambiental sostenible de la región San Martín, 2021. La investigación es de tipo básica, con enfoque cuantitativo, diseño no experimental a nivel descriptivo; a su vez, es proyectiva. La muestra fue constituida por 25 profesionales técnicos y funcionarios del gobierno regional, local y otras organizaciones. Para la recolección de los datos se utilizó la encuesta y el cuestionario dotados de validez y confiabilidad. Se encontró que, el estado situacional de la ecológica es incipiente (64\%), en políticas ambientales (52\%) y respecto al agua limpia y saneamiento (68\%) respectivamente, reflejado en un $56 \%$ de opiniones que consideran al desarrollo ambiental sostenible como incipiente; es decir, en la región 
no hay evidencias de acciones y políticas orientadas al cuidado, conservación y sostenibilidad. En ese sentido, se diseñó una propuesta, concluyéndose que, la sistematicidad del modelo de gestión basado en el ordenamiento territorial contribuye favorablemente al desarrollo ambiental sostenible en la región San Martín; por cuanto, el $40 \%$ de apreciaciones consideran que es muy adecuado, con un promedio de 8.0 puntos con \pm 1.35 desviaciones; es decir, la propuesta según los expertos contiene sistematicidad y coherencia para resolver el problema.

Palabras Clave: Desarrollo; ambiente; sostenible; gestión 


\title{
Territorial planning model for the sustainable environmental development of the San Martín region
}

\begin{abstract}
The present study aimed to determine the situational status of the sustainable environmental development of the San Martín region, 2021. The research is of a basic type, with a quantitative approach, non-experimental design at a descriptive level; in turn, it is projective. The sample was made up of 25 technical professionals and officials from the regional and local government and other organizations. To collect the data, the survey and questionnaire endowed with validity and reliability were used. It was found that the situational state of the ecological is incipient (64\%), in environmental policies (52\%) and regarding clean water and sanitation (68\%) respectively, reflected in $56 \%$ of opinions that consider sustainable environmental development as incipient; In other words, in the region there is no evidence of actions and policies aimed at care, conservation and sustainability. In this sense, a proposal was designed, concluding that the systematic nature of the management model based on land use planning contributes favorably to sustainable environmental development in the San Martín region; because $40 \%$ of the appraisals consider it to be very adequate, with an average of 8.0 points with \pm 1.35 deviations; that is, the proposal according to the experts contains systematicity and coherence to solve the problem.
\end{abstract}

Key Words: development; environment; sustainable; management

Artículo recibido: 30 noviembre. 2021 Aceptado para publicación: 29 diciembre 2021 Correspondencia: pedrocunya@hotmail.com Conflictos de Interés: Ninguna que declarar 


\section{INTRODUCCIÓN}

La sustentabilidad ambiental del avance es una discusión que ha crecido mucho, sin embargo, aún no tiene un acuerdo mundial sobre sus partes y sus implicaciones genuinas, a la luz del hecho de que, si bien las dos ideas comparten las palabras Desarrollo y Sostenibilidad prácticamente hablando, del mismo modo, parecen ser comparativos, no lo son, y su desorden puede conducir sin mucho esfuerzo a errores en la definición del enfoque y sorprendentemente más en su ejecución a través de los procesos de administración.

Este tema no es menor, dado que la primera pregunta que debe responder una Nación que necesita avanzar hacia el Desarrollo Sostenible es definitivamente darse cuenta de lo que se está discutiendo, caracterizando obviamente qué es lo que necesita sustentar en existencia, por ejemplo, asumiendo que es la ley y el orden, asumiendo que es desarrollo monetario, asumiendo que es la satisfacción personal de la población, asumiendo que es la disposición del trabajo natural y los productos de los entornos, o por otro lado asumiendo que es cada uno de ellos en de forma exhaustiva, ajustada e imparcial (Vega, 2015).

El patrón de una condición biológica restablecida de para todos con manejabilidad infiere que la humanidad, los Estados y las organizaciones público-privadas coordinen un trabajo alternativo en la historia del medio ambiente, para dar forma a colusiones que puedan reforzar una economía que se encuentra generosamente en la naturaleza y estructuras de mantenibilidad para las comunidades. sociedad, y que desde los círculos institucionales de la autoridad pública se asegure la prosperidad de la población y de aquellos colectivos subrepresentados a través de estrategias públicas integrales y modelos de administración ecológica (Canaza, 2019).

El cambio climático, en la actualidad, es el problema realmente ecológico que enfrenta la humanidad debido a las salidas consistentes y desiguales de sustancias que agotan la capa de ozono (GEI) por parte de las naciones industrializadas, los ejercicios antropogénicos y entre diferentes maltratos de los activos regulares. Asimismo, se debe al gran efecto de la actividad del hombre sobre la naturaleza y el clima. (Alva, 2019).

El cambio ambiental y la mediación humana expandida están llevando a la Amazonía a un punto de inflexión final, con altos ritmos de deforestación, movimiento y contaminación en el distrito, poniendo en peligro sus sistemas biológicos y afectando a 
las sociedades cercanas. (Orihuela y Rojas, 2017). Y es que La deficiencia de las administraciones estatales cercanas constituye probablemente el mejor obstáculo para un giro razonable de los acontecimientos, razón por la cual es importante ensamblar o fortalecer sus límites institucionales en una administración natural, que debería ser vital del gobierno nacional. La capacidad institucional del gobierno es un atributo de intervención en cuestiones públicas que debiese ayudar a satisfacer las demandas y necesidades del conjunto de la población (Coaquera, 2015).

Para Morales (2002), es necesario buscar avances limpios, enfoques suficientes y permitir la inversión dinámica de los vecinos para lograr una mejora ecológica razonable en el Perú y, en consecuencia, tener la opción de brindar prosperidad y una satisfacción personal superior en un tiempo prolongado.

Por lo tanto, es de suma importancia continuar con los parámetros establecidos y concretar el avance sostenible en el Perú (p. 82). Los problemas ambientales comprenden un aspecto mundial, es decir, abarca todo el planeta, influye en todos los entornos como resultado del avance de la innovación y la industrialización de naciones que buscan el desarrollo monetario, razón por la cual algunas asociaciones financieras globales de Primera línea, como el Banco Mundial y la Organización para la Cooperación y el Desarrollo Económicos (OCDE), afirman estas inquietantes expectativas. Por ejemplo, la OCDE ha introducido un historial extremadamente desalentador de las dificultades que enfrenta la humanidad: luchar contra el cambio ambiental, detener la deficiencia de biodiversidad, proporcionar agua nueva, garantizar una desinfección pública suficiente y disminuir la contaminación del agua. efectos del debilitamiento ecológico en el bienestar de las personas (OCDE, 2011).

Es por ello, que la planificación ambiental ha sido considerada desde su origen como un material en sentido general centrado en peculiaridades regulares de carácter biológico y topográfico, se reconoce progresivamente la incorporación de las cuestiones políticas, monetarias y sociales que va encontrando la población, siendo este el inicio etapa para cualquier preparación y administración ecológica (Rodríguez, 2020). En los años actuales, las selvas amazónicas en Perú muestran una deficiencia acumulada de 10 millones de hectáreas a 1900, lo que aborda un 12\% más bajo en inclusión total debido al hecho de principios de siglo; La investigación de las estadísticas de seguros de la selva amazónica a lo largo de la duración 2001 - 2013 muestra, sin importar las disminuciones 
transitorias, una muestra a cargo de la deforestación que sigue creciendo con una deficiencia regular de 113.000 hectáreas cada año, que superó las ciento cuarenta. , 000 hectáreas dentro de los años 2005, 2009, 2012 y 2013 (Chappa, 2019, p. 32).

En el Perú, en estas últimas décadas la expansión demográfica en los términos de arreglos metropolitanos se ha desarrollado inesperadamente, escapando a los futuros indicadores con respecto al uso de la tierra y el tablero. Los asentamientos rurales han sido descuidados y mal interpretados por expertos y especialistas que han trabajado sin considerar modelos rústicos de avance metropolitano factibles. Este tema no es ajeno a los distritos, que han tenido un avance metropolitano que no considera el escenario metropolitano provincial donde se encuentra este pueblo, lo que ha creado una peculiaridad de degradación en tres partes: corrupción social, degradación del hospedaje y del espacio. público; y escenario y degradación ecológica (Tinajeros, 2015, p. 29).

Para, Hallazi (2017), el desorden territorial se puede diagnosticar cuando surge una guerra, y esta tensión está presente en aproximadamente más del $70 \%$ de los distritos tarde o temprano dentro de la U. S. A., Donde existan conflictos por límites territoriales. La Defensoría del Pueblo en 2017, manifestó 177 conflictos durante el mes de julio de los cuales ciento treinta son de carácter socioambiental (minería, hidrocarburos, energía, agroindustria, silvicultura), y la gran mayoría de esos conflictos confieren superposiciones. Intereses en un espacio territorial idéntico (p. 2-3).

Es por ello, que el ordenamiento regional debe distinguir obviamente los espacios de administración única, una regla principal para lograr el giro económico de los acontecimientos; y solo en este escenario podríamos imaginar ejercicios no degradantes que ayuden a salvar la madera y el clima en general y aceptamos que la industria de viajes con inversión social sería un socio óptimo para su ejecución y mejora. (Coello, 2017)

San Martín en estas últimas décadas, se ha expandido demográfica aceleradamente, y esto se debe a la inmigración de la población de la mayor parte de la sierra norte del Perú, esta situación ha permitido que numerosas familias se asientan en cabeceras de los ríos y realicen la siembra indiscriminada de cultivos generando así la deforestación que afecta, según (López 2019), (Artículo científico).

De la Pontificia Universidad Católica del Perú la deforestación en la Amazonía peruana es una molestia severa que daña a las especies que la habitan, especialmente a las endémicas, debido a que tienen una variedad restringida de distribución. Como en el caso 
de Perú, una de las 3 especies endémicas de primates, el mono tocón de San Martín (Plecturocebus oenanthe), ha sido etiquetado como en grave peligro de extinción debido a amenazas que incluyen la deforestación y el comercio climático.

Por lo tanto, la tarea de mitigar el proceso de deforestación se traduce en la implementación de sistemas de manejo adecuados que sostengan el efecto mínimo sobre la cubierta forestal y las fuentes naturales. De esta manera que es fundamental establecer pautas de mejora relevantes y claras de manera técnica seguidas a través de los respectivos presupuestos para la implementación, ejecución y seguimiento de programas y tareas, mediante una coordinación cercana y articulada entre los sectores involucrados dentro del área, el Gobierno. imperativa, cooperación global y sociedad civil.

Asimismo, es fundamental no olvidar que la deforestación en esta vecindad, como en toda la Amazonía, no solo ha significado la pérdida de especies de madera con costo comercial, sino como alternativa, la pérdida de todo un conjunto de ecosistemas que funcionaban. armoniosa y eficazmente dentro de la vecindad. Naturaleza. Si bien la recuperación de esos es un sistema lento y costoso, está limpio de millas que la asignación esté enfocada a la conservación racional de las áreas boscosas residuales que ocupan el territorio de San Martín, esta premisa y basada totalmente en la ZEE, el boom del suelo de los sistemas productivos (palma, café, cacao, arroz, maíz y otros.

Sin embargo, hay que mencionar que la razón del desarrollo es priorizar el aumento de la productividad de estos sistemas, alejar y desalentar la expansión de las regiones agropecuarias frente al seguro de bosques primarios y secundarios (alejarse de incentivos perversos; en el comercio para que este extra se pueda adquirir bendiciones. Es necesario mencionar que a nivel regional y de los establecimientos que ejecutan paquetes, iniciativas de su campo, hay muchos hechos generados, los cuales deben someterse a una técnica de sistematización y estandarización, para que sea beneficioso y beneficioso, como base para el método de desarrollo local y la implementación y ejecución de proyectos cercanos.

En San Martín, el proceso de planificación del uso de la tierra ha avanzado sustancialmente, especialmente en términos de zonificación financiera (ZEE). Así, la macro SEZ (grado cercano), permitida por debajo de la ordenanza regional No. 012 2006 - GRSM / CR y sus respectivas normas, mediante el cercano decreto No. 002 - 2009 - GRSM / PGR. Con base en esto, proyectos provinciales y distritales o incluso en etapa 
de cuenca en cuanto a la adecuada administración del territorio, se habían realizado y realizado bajo una técnica dinámica y de un acuerdo entre autoridades regionales, sociedad civil y diferentes actores, con el aval de las autoridades críticas.

Esto se ha traducido en la aprobación, en una consulta del Consejo Regional de Política Territorial Regional de San Martín (7 de septiembre de 2012). En el marco de su aprobación, la articulación de los Planes de Ordenamiento Territorial, Planes de Acondicionamiento del Territorio, Planes de Desarrollo Urbano, Planes de Desarrollo Concertado (coordinación con el rango de precios Regional y elaboración de planes de control y otros a nivel regional con base fundamentalmente en la ZEE la territorial sostenible cobertura articulando e integrando la mejora de enfoques y la implementación de artilugios de gestión entre las etapas de gobierno, sociedad civil organizada y el barrio personal, pensando en la infección que impacta los planes de ordenamiento territorial y desarrollo ambiental.

Desarrollo sustentable del entorno. y Un modelo de control ambiental integra una serie de movimientos o estrategias orientadas a la dirección o manejo de los recursos naturales, en un esfuerzo por contribuir al desarrollo sustentable de un territorio, por lo que todas las acciones que se planifiquen y ejecuten, serán buscando realzar la excelencia de la vida de la población, a través de la gestión de técnicas para salvarlo y reducir los malos resultados en los activos herbales del lugar.

Y frente a todo lo expuesto, surge la formulación del problema general:

- ¿Cuál es el estado situacional del desarrollo ambiental sostenible de la región San Martín 2021?

\section{Asimismo, se plantea las siguientes preguntas específicas:}

- ¿Cuál es el estado situacional de la dimensión ecológica del desarrollo ambiental sostenible de la región San Martín 2021 ?

- ¿Cuál es el estado situacional de las políticas ambientales del desarrollo ambiental sostenible de la región San Martín 2021?

- ¿Cuál es el estado situacional del agua limpia y saneamiento del desarrollo ambiental sostenible de la región San Martín 2021 ?

- ¿De qué manera la sistematicidad de un modelo de gestión basado en el ordenamiento territorial contribuye a la mejora del desarrollo ambiental sostenible en la región San Martín 2021? 
- ¿Cuál es la percepción de los expertos sobre la contribución del modelo de gestión basado en el ordenamiento territorial para que contribuya al desarrollo ambiental sostenible en la región San Martín 2021.

Esta investigación tuvo como justificación por conveniencia, porque permitió mejorar la adecuada funcionabilidad del desarrollo ambiental Sostenible en la zona de San Martín a través de los gobiernos vecinos de turno que deben ofrecer un sistema ecológicamente sano, económicamente viable y socialmente honesto. Promover la protección a largo plazo del medio ambiente y sus habitantes; y asegurarse de que las interacciones llevadas a cabo con fuentes naturales ganen de una manera única o cada otra a las afectadas dentro de los sistemas efectivos. Tiene relevancia social, porque sus resultados y conclusiones benefician directamente a instituciones y gobiernos de turno encargadas de plantear lineamientos estratégicos en el desarrollo ambiental sostenible desde un contexto de ordenamiento territorial, donde la propuesta brindada por el autor contribuirá a mejorar la gestión de una manera eficiente y eficaz.

Además, presenta valor teórico, porque la investigación buscó mediante la aplicación de la teoría y los conceptos básicos sobre gestión territorial, ordenamiento territorial y gestión ambiental; encontrar situaciones que afectan su eficacia en la adecuada administración del desarrollo ambiental sostenible de la región San Martín por parte de diversas instituciones y gobiernos locales que intervienen en este campo, de esta manera el investigador pueda contrastar situaciones con la realidad. Tiene implicancias prácticas porque la investigación describió la realidad existente referente a la gestión actual de los procesos de ordenamiento territorial ambiental de la región San Martín junto al proceso de ejecución del desarrollo ambiental sostenible de los recursos utilizados por la población; identificando así las debilidades que hacen que se proceda a un mal funcionamiento del mismo y así poder trazar opciones de solución que permitan generar beneficios en cuanto a una adecuada administración de los espacios territoriales de la Región y del uso sostenible de los recursos naturales. Finalmente, la utilidad metodológica para la presente investigación fue diseñar y validar técnicas e instrumentos para la recolección de la información, que serán un factor clave para el desarrollo los objetivos propuestos, permitiendo tener un mayor alcance en nuestra labor al plantear estrategias adecuadas en cuanto al problema de investigación planteado. 


\section{Como objetivo general de la investigación se plantea:}

- Determinar el estado situacional del desarrollo ambiental sostenible de la región San Martín.

\section{Asimismo, se plantean objetivos específicos como:}

- Diagnosticar el estado situacional de la dimensión ecológica del desarrollo ambiental sostenible de la región San Martín 2021,

- Diagnosticar el estado situacional de las políticas ambientales del desarrollo ambiental sostenible de la región San Martín 2021,

- Diagnosticar el estado situacional del agua limpia y saneamiento del desarrollo ambiental sostenible de la región San Martín 2021,

- Diseñar un modelo de gestión relacionado de modo que contribuya al modelo de gestión basado en el ordenamiento territorial la mejora del desarrollo ambiental sostenible en la región San Martín, 2021,

- Evaluar mediante juicio de expertos la contribución del modelo de gestión basado en el ordenamiento territorial para el desarrollo ambiental sostenible en la región San Martín, 2021.

\section{La hipótesis general que se establece para esta investigación es,}

- HG: El estado situacional del desarrollo ambiental sostenible de la región San Martín 2021 es avanzado.

\section{En cuanto a las hipótesis específicas se tiene que,}

- H1 El estado situacional de la dimensión ecológica del desarrollo ambiental sostenible de la región San Martín 2021, es avanzado.

- H2 El estado situacional de las políticas ambientales del desarrollo ambiental sostenible de la región San Martín 2021, es avanzado.

- H3 El estado situacional del agua limpia y saneamiento del desarrollo ambiental sostenible de la región San Martín 2021, es avanzado.

- H4 La sistematicidad de un modelo de gestión basado en el ordenamiento territorial, aportaran al desarrollo ambiental sostenible en la región San Martín 2021.

- H5 Las contribuciones del modelo de gestión basado en el ordenamiento territorial aportaran al desarrollo ambiental sostenible en la región San Martín 2021, es muy adecuada y excelente contribución, según el juicio de los expertos. 


\section{ESTRATEGIAS METODOLÓGICAS O MATERIALES Y MÉTODOS}

\subsection{Tipo y diseño de investigación}

\section{Tipo de investigación}

Según su finalidad fue de tipo básica con un enfoque cuantitativo, debido a que se aplicó conocimiento e información de las investigaciones básicas (Alvitres, 2000). Igualmente, la investigación es de tipo proyectiva porque propone una solución del problema. Al respecto, Hurtado (2008) señala que un estudio es proyectivo, cuando propone soluciones a una determinada situación partiendo de una indagación, exploración, descripción, explicación y propone alternativas de cambio, pero, no necesariamente se ejecutan.

\section{Diseño de investigación}

Según Concytec (2018), se empleó el diseño no experimental a nivel descriptivo y proyectivo, para realizar un diagnóstico de la realidad de la situación actual de la problemática, se analizó. Según Chávez (2007) el diseño no experimental permite observar los fenómenos y cómo estos se desarrollan en su estado natural; sin la manipulación de las variables. Cuyo esquema es el siguiente:

\section{$\mathbf{M} \mathbf{O}$}

\section{Dónde:}

$\mathrm{M}=$ Muestra de la investigación.

$\mathrm{O}=$ Desarrollo ambiental sostenible

\subsection{Variables y operacionalización}

- Variable 1: Desarrollo ambiental sostenible

- Variable 2: Modelo de gestión basado en el ordenamiento territorial.

2.3 Población (criterios de selección), muestra, muestreo, unidad de análisis

\section{Población}

La población estuvo conformada por 40 profesionales vinculados a la gerencia de desarrollo ambiental del gobierno regional y local; así como, de organizaciones no gubernamentales que intervienen en la región San Martín. Según García (2018), son todos los elementos (personas, objetos, organismos, historias, informes) que participan del fenómeno que fue definido y delimitado en el análisis del problema de investigación. En ese sentido, para diagnosticar el estado situacional del desarrollo ambiental sostenible, se coordinó el acceso a las percepciones de los especialistas en temas referidos a la gestión 
y desarrollo ambiental. Igualmente, para evaluar la propuesta se acudió a profesionales expertos que ostentan posgrado a nivel de doctorado y conocen el la problemática y solución ambiental, a fin de valorar la sistematicidad de la propuesta.

\section{Criterios de inclusión}

- Profesionales y/o especialista que residan en las diez provincias de la región San Martin con disposición para opinar.

- Profesionales y/o especialistas con experiencia laboral y conocimiento sobre el problema de investigación.

\section{Criterios de exclusión}

- Profesionales y/o especialistas que no residen en la región de San Martín, 8 expertos en temas medio ambientales y 2 expertos en ordenamiento territorial.

- Profesionales y/o especialistas que no deseen formar parte del estudio, 5 expertos en medios ambientales.

\section{Muestra}

La muestra según García (2018) es un subconjunto representativo de un universo o población. Es representativo cuando refleja fielmente las características de la población. En este caso, para recolectar las opiniones y describir el estado situacional del desarrollo ambiental sostenible de la región San Martín, la muestra estuvo conformada por 25 profesionales, entre técnicos, ingenieros y funcionarios vinculados con el desarrollo ambiental y que laboran en instancias del gobierno regional y local; así como, de algunas organizaciones implicadas con el medio ambiente; tale como: Gobierno Regional (GR), Proyecto Alto Mayo (PEAM), Autoridad Nacional del Agua - MINAGRI SM (ANA), Proyecto Especial Bajo Mayo y Huallaga Central (PEBMyHC), Gobiernos Locales (GL) y Autoridad Regional del Agua (ARA)

\section{Muestreo}

Por la naturaleza de una población pequeña y según los criterios de inclusión y exclusión, se utilizó el muestreo no probabilístico y selección por conveniencia; al respecto, Navas (2017) precisa que, este tipo de estudios no hacen uso de un medio estadístico, para la aceptación de la totalidad de la población. Por lo contrario, son determinadas a intención del investigador. 


\section{Unidad de análisis}

Cada profesional o especialista que participa respondiendo el cuestionario para describir el estado actual del desarrollo sostenible de la región San Martín.

\subsection{Técnicas e instrumentos de recolección de datos, validez y confiabilidad}

Técnicas. La técnica empleada fue la encuesta, a través del cual se elaboró un instrumento con una serie de preguntas relacionadas directamente con las dimensiones e indicadores de la variable de estudio.

\section{Instrumentos de recolección de datos}

Se utilizó un cuestionario, elaborado por el autor, con un conjunto de ítem acorde a los indicadores y dimensiones de la variable. Consta de 49 preguntas distribuidas en las tres dimensiones.

\begin{tabular}{llc} 
Dimensiones & Ítems & Opciones de respuesta \\
\hline Ecológica & Del 01 al 11 & Muy malo 1 \\
Políticas ambientales & Del 12 al 40 & Malo 2 \\
\multicolumn{1}{c}{ Agua limpia y } & Del 41 al 49 & Regular 3 \\
\multicolumn{1}{c}{ saneamiento } & & Bueno 4 \\
\hline
\end{tabular}

A partir del puntaje máximo y puntaje mínimo, se elaboró una escala ordinal con rangos Validez

El referido instrumento fue sometido al juicio de cinco expertos, quienes han revisado la claridad, coherencia, cantidad, intencionalidad, pertinencia, etc., de cada uno de los ítems con respecto a los indicadores y dimensiones de la variable; luego han realizado observaciones y comentarios; así como, valoraciones cuantitativas en un formato de informe; los cuales fueron tenidos en cuenta para mejorar el cuestionario.

Valoración de juicio de expertos

\begin{tabular}{lccccc}
\hline Variable & N. $^{\circ}$ & Especialidad & $\begin{array}{c}\text { Promedio de } \\
\text { validez }\end{array}$ & \multicolumn{2}{c}{ Opinión del experto } \\
\hline Desarrollo & 1 & Metodólogo & 4,5 & Es & coherente y \\
procede su aplicación \\
ambiental & 2 & Metodólogo & 4,9 & & Es aplicable. \\
sostenible & 3 & Metodólogo & 4,8 & Es aplicable \\
& 4 & Especialista & 4,7 & Es aplicable \\
& 5 & Especialista & 4,2 & Es aplicable \\
\hline
\end{tabular}


La confiabilidad es la consistencia interna del instrumento, el cual se estima o calcula mediante el alfa de Cronbach; según Welch \& Comer (1988), cuánto más cerca se encuentre el valor del alfa a 1 mayor es la consistencia interna de los ítems analizados. Para este caso, la confiabilidad fue calculada con las valoraciones cuantitativas expresadas en el informe de expertos, respecto a cada uno de los diez criterios, habiéndose obtenido una confiabilidad alta de 0,72 , tal como se precisa en los anexos.

\subsection{Procedimientos}

En la fase diagnóstica o primer momento, luego de haberse identificado el problema de investigación y por consiguientemente formulado el problema científico, se procedió a planificar e implementar el diagnóstico en el contexto de la región, a fin de corroborar los indicios del poco o incipiente desarrollo ambiental sostenible. Para ello, se operacionalizó la variable, a partir de los indicadores se elaboró ítem y configuró un cuestionario. Se seleccionó e identificó la muestra y aplicó el instrumento, combinando estrategias; vía telefónica, otro correo electrónico y en forma presencial. Los datos fueron procesados, analizados e interpretados; corroborándose el incipiente desarrollo ambiental sostenible de la región San Martín; estas evidencias estadísticas, más las experiencias del suscrito, conllevó a la necesidad de edificar una propuesta denominada Modelo de gestión basada en el ordenamiento territorial.

En la fase proyectiva o de diseño, se procedió a indagar otras experiencias investigativas y propuestas para afrontar esta problemática, en perspectiva de tener un referente; así como, se revisó los lineamientos de política ambiental regional y el marco normativo sobre el desarrollo ambiental, centrándose en el ordenamiento territorial como como estrategia de desarrollo. Igualmente, se consultó las principales teorías que explican y describen el desarrollo, el medio ambiente, los sistemas y ecosistemas. Se tuvo en cuenta el lineamiento de la UCV en relación a los componentes del modelo y se organizó un primer bosquejo de modelo. Luego con el aporte de otros profesionales se fue diseñando la propuesta, teniendo en cuenta cómo contribuir a la mejora del desarrollo ambiental para la región San Martín.

En la fase de consulta a los expertos en gestión pública y especialistas en materia ambiental, se les solicitó su opinión, observaciones y sugerencias sobre el diseño del modelo de gestión basado en el ordenamiento territorial para mejorar el desarrollo ambiental; en perspectiva de recoger sus aportes. Para ello, se utilizó un cuestionario 
sugerido por la UCV y cuyas opiniones y valoraciones fueron procesadas y analizadas, dotándole de sistematicidad al modelo y consecuente aval técnico respecto a su pertinencia en la contribución para coadyuvar en la solución del problema de investigación.

\subsection{Método de análisis de datos}

Posterior a la recolección de datos, se procedió a realizar el vaciado de información en Excel, las cuales fueron organizadas en tablas y analizadas mediante técnicas descriptivas como la frecuencia, porcentaje, media aritmética, cuyos resultados se analizaron e interpretaron acorde con los objetivos e indicadores de cada dimensión de las variables y opiniones de los expertos.

\subsection{Aspectos éticos}

En todo momento, el estudio fue desarrollado por iniciativa del investigador, la misma que está basada en los principios de autonomía, beneficencia, no maleficencia y justicia. Con respecto al principio de autonomía, se tuvo presente que los resultados que se obtuvo en la investigación servirán de herramienta en la toma de decisiones o presente que para desarrollar la investigación se contó con la autorización de las instituciones donde se hizo el estudio, por ello se pudo realizar la investigación.

En relación al principio de beneficencia, se tuvo presente que los resultados que se obtuvo en la investigación servirán de herramienta en la toma decisiones de la institución donde se realizó el estudio.

En referencia al principio de no maleficencia, el estudio realizado no causa daño a los participantes, además de ello se garantizó la confidencialidad de sus datos personales y sirvió para la preparación de la investigación.

Acerca del principio justicia, se aseguró que los aportes de la investigación van tener impacto positivo en la población de la región San Martín

En todo momento, se respetó el principio de la propiedad intelectual, haciendo uso de las citas y referencias basados en la norma APA $7^{\circ}$ edición, con el fin de salvaguardar los derechos de autor y que los datos obtenidos no sean considerados plagio. Así también se trabajará con autonomía, pues la información se redactará bajo el criterio y experiencias propias de los involucrados, demostrando una posición neutral a cualquier información o comentario realiza por algunos autores 


\section{RESULTADOS Y DISCUSIÓN}

\section{Tabla 1}

Nivel de desarrollo ambiental sostenible en relación a la dimensión ecológica - región San Martin, 2021

\begin{tabular}{lcccc}
\hline Nivel & & & Cantidad & Porcentaje \\
\hline Incipiente & 11 & 33 & 16 & $64 \%$ \\
Moderado & 34 & 44 & 9 & $36 \%$ \\
Avanzado & 45 & 55 & 0 & $0 \%$ \\
& & & 25 & $100 \%$ \\
\hline
\end{tabular}

Fuente: Datos obtenidos del cuestionario

Se aprecia que la mayoría de los entrevistados (64\%) manifiestan que el desarrollo ambiental sostenible en relación a la dimensión ecológica en la región San Martin es incipiente. En efecto, solamente existes escasas acciones estratégicas orientadas diversidad biológica, animales silvestres en peligro de extinción, plantas forestales en peligro de extinción, plantas medicinales en, peligro de extinción, contaminación hídrica, contaminación del suelo, contaminación acústica, contaminación lumínica, contaminación visual, contaminación térmica, contaminación atmosférica la control y manejo del agua, bosque, animales; así como, para minimizar la contaminación del aire, el suelo.

\section{Tabla 2}

Nivel de desarrollo ambiental sostenible en relación a la dimensión Políticas ambientales - región San Martin, 2021

\begin{tabular}{lcccc}
\hline Nivel & & & Cantidad & Porcentaje \\
\hline Incipiente & 29 & 87 & 13 & $52 \%$ \\
Moderado & 88 & 116 & 12 & $48 \%$ \\
Avanzado & 117 & 145 & 0 & $0 \%$ \\
& & & 25 & $100 \%$ \\
\hline
\end{tabular}

Fuente: Datos obtenidos del cuestionario

Se puede señalar que el (52\%) de los consultados determinaron que la dimensión de Políticas Ambientales en la región San Martin es incipiente. En consecuencia, la correlación de las capacidades sobre programas de reforestación, conservación de cuencas hidrográficas, conservación y manejo forestal, contaminación ambiental. 


\section{Tabla 3}

Nivel de desarrollo ambiental sostenible en relación a la dimensión Agualimpia y

Saneamiento - región San Martin, 2021

\begin{tabular}{lcccc}
\hline Nivel & & & Cantidad & Porcentaje \\
\hline Incipiente & 9 & 27 & 17 & $68 \%$ \\
Moderado & 28 & 36 & 8 & $32 \%$ \\
Avanzado & 37 & 45 & 0 & $0 \%$ \\
& & & 25 & $100 \%$ \\
\hline
\end{tabular}

Fuente: Datos obtenidos del cuestionario

Se estima que la mayoría de los encuestados (68\%), muestran que el desarrollo ambiental sostenible a la dimensión de agua limpia y saneamiento, consideraron incipiente. Lo cual se puede observar en los resultados teniendo como indicadores, agua limpia y saneamiento, acceso al agua potable, acceso a saneamiento e higiene, calidad de agua, contaminación del agua, aguas residuales, uso eficiente de recursos hídricos, ecosistemas relacionados con agua, y la participación de las comunidades locales

\section{Tabla 4}

Nivel de desarrollo ambiental sostenible - región San Martín, 2021

\begin{tabular}{lcccc}
\hline & & & Cantidad & Porcentaje \\
\hline Incipiente & 49 & 147 & 14 & $56 \%$ \\
Moderado & 148 & 196 & 11 & $44 \%$ \\
Avanzado & 197 & 245 & 0 & $0 \%$ \\
& & & 25 & $100 \%$ \\
\hline
\end{tabular}

Fuente: Datos obtenidos del cuestionario

En la tabla 4, se observa que el 56\% de los encuestados (14) consideran que el desarrollo ambiental sostenible en la región San Martín es incipiente; es decir, no hay políticas ambientales en relación a la diversidad ecológica, ni en saneamiento de las ciudades y la población. 


\section{Tabla 5}

Opiniones de los expertos sobre la sistematicidad del modelo de gestión basado en el ordenamiento territorial

\begin{tabular}{|c|c|c|c|c|c|c|c|c|c|c|}
\hline \multicolumn{2}{|c|}{ Aspectos a valorar del Modelo } & 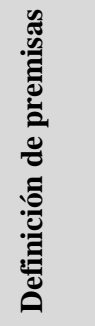 & 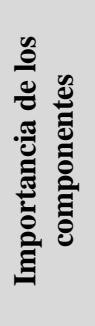 & 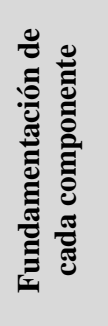 & 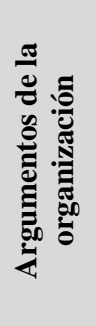 & 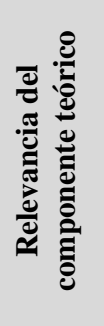 & 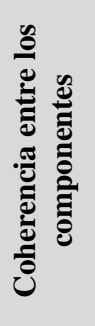 & 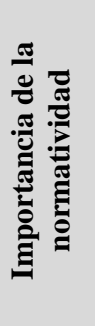 & 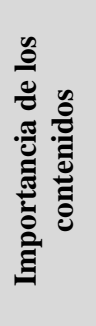 & 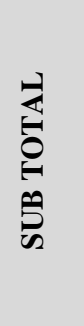 \\
\hline \multirow{2}{*}{ Muy adecuado } & Cantidad & 3 & 3 & 2 & 1 & 2 & 0 & 3 & 2 & 16 \\
\hline & Porcentaje & $60 \%$ & $60 \%$ & $40 \%$ & $20 \%$ & $40 \%$ & $0 \%$ & $60 \%$ & $40 \%$ & $40 \%$ \\
\hline \multirow{2}{*}{ Bastante adecuado } & Cantidad & 1 & 1 & 3 & 1 & 1 & 5 & 1 & 2 & 15 \\
\hline & Porcentaje & $20 \%$ & $20 \%$ & $60 \%$ & $20 \%$ & $20 \%$ & $100 \%$ & $20 \%$ & $40 \%$ & $38 \%$ \\
\hline \multirow{2}{*}{ Adecuado } & Cantidad & 1 & 1 & & 3 & 1 & 0 & 1 & 1 & 8 \\
\hline & Porcentaje & $20 \%$ & $20 \%$ & $0 \%$ & $60 \%$ & $20 \%$ & $0 \%$ & $20 \%$ & $20 \%$ & $20 \%$ \\
\hline \multirow{2}{*}{ Poco adecuado } & Cantidad & 0 & 0 & 0 & 0 & 1 & 0 & 0 & 0 & 1 \\
\hline & Porcentaje & $0 \%$ & $0 \%$ & $0 \%$ & $0 \%$ & $20 \%$ & $0 \%$ & $0 \%$ & $0 \%$ & $3 \%$ \\
\hline \multirow{2}{*}{ Inadecuado } & Cantidad & 0 & 0 & 0 & 0 & 0 & 0 & 0 & 0 & 0 \\
\hline & Porcentaje & $0 \%$ & $0 \%$ & $0 \%$ & $0 \%$ & $0 \%$ & $0 \%$ & $0 \%$ & $0 \%$ & $0 \%$ \\
\hline \multirow{2}{*}{ TOTAL } & Cantidad & 5 & 5 & 5 & 5 & 5 & 5 & 5 & 5 & 40 \\
\hline & Porcentaje & $100 \%$ & $100 \%$ & $100 \%$ & $100 \%$ & $100 \%$ & $100 \%$ & $100 \%$ & $100 \%$ & $100 \%$ \\
\hline
\end{tabular}

Fuente: Datos obtenidos del cuestionario

En la tabla 5, se estima las opiniones cuantitativas sobre los diversos aspectos del Modelo de Gestión Basado en el Ordenamiento para el Desarrollo Ambiental Sostenible en la Región San Martín 2021, observándose que el 40\% de apreciaciones (16) de los expertos consideran que la propuesta es muy adecuada, un $38 \%$ de opiniones (15) califican que es bastante adecuada, un $20 \%$ de valoraciones (8) califican adecuado mientras que $3 \%$ de estimaciones (1) califican poco adecuado. En consecuencia, se deduce que la mayoría de las opiniones coinciden que, el modelo refleja sistematicidad y coherencia en relación a la premisa, importancia y fundamentación de los componentes, los argumentos de su organización, así como, la normatividad y contenidos son esenciales y consistentes, de la misma manera la relevancia del componente teórico.

\section{DISCUSIÓN}

De acuerdo a los resultados obtenidos de la aplicación de los instrumentos se evidencia que la situación actual del Ordenamiento Territorial en relación sobre el desarrollo 
ambiental sostenible es incipiente en las tres dimensiones analizados, resultados, como la dimensión ecológica, con 56\% es incipiente, la dimensión de políticas ambientales de $52 \%$ y también la dimensión de agua limpia y saneamiento de un 68\%, lo cual evidencia deficiencias en la gestión de desarrollo ambiental en correlación al ordenamiento territorial, tales como la deforestación, perdidas de ecosistemas, crecimiento desordenado en las zonas urbanas y rurales que no cuenta con los servicios agua y alcantarillados y luz eléctrica, lo cual hace sensible a cumplir con las normas por condiciones electorales en un bien de desarrollos de bienes de consumo productivo y servicios que se le otorga a los ciudadanos.

El proceso de descentralización tiene que ser puesto en agenda por los gobiernos locales para superar las limitaciones y avanzar en el proceso lo cual implica poder desarrollar políticas orientadas a lograr una mayor cohesión social y territorial, un uso y ocupación racionales, sostenibles y democráticos del territorio, y una dinámica institucional que supere la intervención desordenada y sectorial que hoy prima en el estado. Dichos resultados guardan relación con Hallazi (2017), donde concluyo el desorden territorial puede ser identificado cuando nace un conflicto, y esta tensión se encuentra presente en aproximadamente más del $70 \%$ de los distritos de todo el país, donde existen conflictos por los límites territoriales.

Del mismo modo guarda relación con la investigación realizada por Vega, L. (2015), quien Concluyó: que en términos de sostenibilidad significará que el desarrollo sostenible debe ser ambientalmente sostenible, para garantizar en tiempo y espacio la dotación de recursos naturales y de servicios ambientales, y por otro, que las actividades humanas sean realizadas en armonía con las leyes de los sistemas naturales de tal manera que se preserve la integridad de los procesos que rigen los flujos de energía, materia y biodiversidad de los ecosistemas.

Lograr que el desarrollo sea ambientalmente sostenible o mejor, lograr la sostenibilidad ambiental del desarrollo será competencia directa del ente rector de la política y de la gestión ambiental de cada país, siempre de manera coordinada entre el sector público, el sector económico y la sociedad civil.

Por otro lado, la correlación de los resultados con el ordenamiento territorial se sustenta en el conocimiento y la investigación de la diversidad del territorio y la sostenibilidad de sus ecosistemas; en la articulación intergubernamental e intersectorial; en el fomento de 
iniciativas de inversión pública y privada; y en la promoción del diálogo, la participación ciudadana y la consulta previa a los pueblos indígenas u originarios. Se desarrolla sobre la base de la articulación del nivel intergubernamental e intersectorial a través de espacios que promueva la cohesión, integración y cooperación entre el nivel regional, provincial y distrital, tales como las comisiones técnicas de Zonificación Ecológica Económica-ZEE y la comisión técnica de los instrumentos técnicos sustentatorios del ordenamiento territorial, cabe mencionar que también se considera la participación de los sectores ya que resulta necesario coordinar y planificar la intervención de manera estratégica sobre el territorio (MINAM, 2016, p. 8-9).

Dichos resultados son también expresados por el proceso de ordenamiento territorial, los gobiernos regionales y locales son responsables de la elaboración de sus instrumentos técnicos sustentatorios, para lo cual deberán considerar las siguientes etapas de desarrollo, en concordancia con la Resolución Ministerial N²135-2013-MINAM y demás normativa vigente en esta materia. i) Preparación: En esta etapa se definen los objetivos y estrategias a seguir en el proceso de ordenamiento territorial, promoviendo su articulación y vinculación con el desarrollo del ámbito geográfico en estudio. ii) Diagnóstico: comprende la generación de información que permita identificar diferentes condiciones que presenta el territorio desde un enfoque sistémico e integrador, así como el análisis de las diferentes dinámicas territoriales. iii) Formulación y aprobación del plan: Comprende la elaboración del POT, para lo cual se deberá definir la visión de desarrollo, las políticas y estrategias -objetivos, programas, proyectos y acciones. iv) Implementación del POT: Corresponde la ejecución de actividades identificadas en el POT. v) Monitoreo: Comprende el desarrollo de acciones de manera permanente y orienta la fase de implementación en corto, mediano y largo plazo, promoviendo el cumplimiento de las estrategias (MINAM, 2016, p.13-14).

Asimismo guardan relación Valdez (2020) considera que los efectos que provoca el hombre, tienen una relación directa con su medio, así que estas fuerzas son reflejo de su accionar, como el cambio climático, que día a día se incrementa, además de esto, la contaminación de los océanos y destrucción de capa de ozono. Solo el hombre cuando sea consecuente con sus acciones podrá detener los impactos negativos que perjudican al medio ambiente y a la vez a su salud (p. 15). Dichos resultados guardan relación La gestión ambiental es sumamente importante, ya que se encargar en gestionar de manera 
eficiente los recursos, herramientas y acciones pertinentes para la mejora del medio ambiente y la sociedad que lo habita. Para Ruiz (2018) la importancia de la gestión ambiental implica un conjunto de medidas que busca minimizar los impactos negativos que pueden afectar al medio ambiente, asimismo se debe establecer el equilibrio entre ambos aspectos (p. 39). Põder (2006) mencionó que se debe evaluar el impacto sobre el medio ambiente y considerar las consecuencias que repercutirá, es así que estos impactos ambientales generarán a futuro, nuevas políticas de transformación que favorecerán los proyectos de desarrollo sostenible en el medio. Es así que se debe mantener una estrategia adecuada que podrá medir las actividades significativas que repercutirán a futuro (p. 735). También se relaciona con la gestión ambiental, agrupa las diversas actividades o estrategias, que se pueden desarrollar en aras de la protección ambiental y prevenir conflictos ambientales. Se basa en conocer el "que hay que hacer", para brindar protección y conservación del ambiente, saber dar uso adecuado a los recursos que nuestro mundo nos ofrece, y así lograr un equilibrio apropiado para el desarrollo sostenible Cordero, (2004).

\section{CONCLUSIONES Y CONSIDERACIONES FINALES}

- La sistematicidad del modelo de gestión basado en el ordenamiento territorial contribuye favorable al desarrollo ambiental sostenible en la región San Martín, 2021; por cuanto, se obtuvo una media de 8.0 puntos, con una desviación estándar \pm 1.35 desviaciones respecto a la media; es decir, la propuesta según los expertos contiene sistematicidad y coherencia interna para resolver el problema.

- El estado situacional de la dimensión ecológica del desarrollo ambiental sostenible de la región San Martín 2021, es incipiente, según el 64\% de opiniones; es decir, existen muy pocas acciones estratégicas orientadas a conservar la diversidad biológica.

- El estado situacional de las políticas ambientales del desarrollo ambiental sostenible de la región San Martín 2021, es avanzado.

- El estado situacional del agua limpia y saneamiento del desarrollo ambiental sostenible de la región San Martín 2021, es avanzado

\section{REFERENCIAS BIBLIOGRAFICAS}

Alvitres, V. (2000). Método Científico, Planificación de la Investigación (Editorial Ciencias (ed.)). 
Alva, W. (2019). Ecoeficiencia: Nueva estrategia para la educación ambiental en instituciones educativas. Investigación Valdizana, 13(2), 77-84. https://doi.org/10.33554/riv.13.2.233

Arias, R. I., González, R., Herrera, A., \& Demesio, R. (2015). Indicadores ambientales en comunidades kichwa amazónicas ecuatorianas para elaborar una estrategia de desarrollo sostenible. Centro Agrícola, 42(2), 71- 78. http://cagricola.uclv.edu.cu/descargas/pdf/V42-Numero_2/cag10215.pdf

Benete, A. (2015). El desarrollo rural territorial: Las reservas de biosfera como oportunidad para el desarrollo sostenible. Cooperativismo y Desarrollo (COODES), 3(1), 84-99.

https://dialnet.unirioja.es/descarga/articulo/5294663.pdf

Bernal, A. L. (2018). Seminario internacional de ecología urbana. "Ordenamiento de las ciudades y gestión empresarial para la sostenibilidad”. Revista Proyección Social, 1(1), 54-57.

https://journalusco.edu.co/index.php/rps/article/view/1562

Canaza, F. A. (2019). De la educación ambiental al desarrollo sostenible: desafíos y tensiones en los tiempos del cambio climático. Rev. Ciencias Sociales Universidad de Costa Rica, 165(3),155-172.

https://revistas.ucr.ac.cr/index.php/sociales/article/download/40070/40696/

Castillo, G. (2019). Contaminación de canales de agua lluvia en zonas urbanas. (Tesis de maestría). Universidad Santiago de Cali, Colombia. http://repository.usc.edu.co/handle/20.500.12421/4622

Castro, E. (2015). Panorama regional del desarrollo sostenible en América Latina. Luna Azul, 40, 195-212. https://doi.org/10.17151/luaz.2015.40.13

Chappa, C. E. (2019). Valoración económica del servicio ambiental por secuestro de carbono en la biomasa aérea de diferentes sistemas agroforestales - en la Región San Martín - Perú. (Tesis de doctorado). Universidad Nacional de San Martín, Tarapoto, Perú. http://hdl.handle.net/11458/3342

CEPAL. (2013). Desarrollo sostenible en América Latina y el Caribe. Seguimiento de la agenda de las Naciones Unidas para el desarrollo post-2015 y Río+20. Bogotá, Colombia: Comisión Económica para América Latina y el Caribe. 
Ceuta Espacio Verde. (2018). Especiales ambientales CONCIENCIA AMBIENTAL. Lineaverdeceutatrace.Com. http://www.lineaverdeceutatrace.com/lv/consejosambientales/conciencia-ambientual/Que-es-el-desarrollo-sostenible.asp

Coaquera, H. M. (2015). Evaluación de la gestión ambiental en las municipalidades distritales de la Provincia de Tacna, periodo: 2009-2010 y el desarrollo sostenible. Propuesta de una auditoría ambiental como instrumento para el planeamiento urbano sostenible. (Tesis de maestría). Universidad Nacional Jorge Basadre Grohmann, Tacna, Perú.

http://repositorio.unjbg.edu.pe/handle/UNJBG/1035

Coello, F. E. (2017). Planificación territorial de espacios rurales para la utilización del turismo como herramienta para la conservación de la riqueza natural y patrimonial de éstos. (Tesis doctoral). Universitat de les Illes Balears, España. http://hdl.handle.net/11201/148217

Cordero, M. B. (2004). Gestión ambiental: Camino al desarrollo sostenible. San José, Costa Rica: Editorial EUNED.

Friedmann, J. y C. Weaver. (1979). Territory and Function, Edward Arnold, London

Gastañaga M, (2018) Agua, saneamiento y salud. Rev Peru Med Exp Salud Publica. 2018;35(2):181-2. doi: 10.17843/rpmesp.2018.352.3732

George, D., \& Mallery, P. (2003). SPSS for Windows step by step: A simple guide and reference. 11.0 update (4thed.). Boston: Allyn \& Bacon

Hallazi, L. (2017). Desorden territorial, conflictos sociales y promoción de inversiones.

ALAI. https://www.alainet.org/es/articulo/187582

Heredia, B. (2020). Gestión de residuos sólidos y desarrollo sostenible en la Municipalidad Distrital de Asia, 2020. (Tesis de maestría). Universidad César Vallejo, Lima, Perú. https://hdl.handle.net/20.500.12692/48536

Larios F (2015) Revista de la Facultad de Ingeniería de la USIL Vol. 2, No 2. Segundo semestre 2015. pp. 09-25

Lopez, K. (2019). Análisis espacial (1987-2017) y predictivo (2050) del hábitat del mono tocón, PLECTUROCEBUS OENANTHE Thomas, en la región San Martín, Perú, Espacio y Desarrollo N³3, 2019, pp. 143-166 (ISSN 1016- 9148) Lima, Perú. https://doi.org/10.18800/espacioydesarrollo.201901.007 
Ministerio del Ambiente - (MINAM). (2015). Ordenamiento territorial para el desarrollo sostenible. https://sinia.minam.gob.pe/download/file/fid/39053

Ministerio del Ambiente - MINAM. (2016). Instrumentos técnico normativos del ordenamiento territorial.

http://download1654.mediafire.com/ybca1bxqpt8g/wxwda528cyuqn2s/Instru mentos+Te\%CC\%81 cnico+Normativos+del+Ordenamiento+Territorial.pdf

Ministerio del Ambiente - (MINAM). (2017). Ley No 28611 - Ley General del Ambiente. Ley $\mathrm{N}^{\circ}$ 28245, Ley Marco del Sistema Nacional de Gestión Ambiental. https://cdn.www.gob.pe/uploads/document/file/473242/DECRETO_SUPRE MO_N\%C2\%BA_008-2005-PCM.pdf

Mendoza, A. (2011). Colombia: Teoría del ordenamiento. Perspectiva Geográfica, 1(7), 161-166. https://doi.org/10.19053/01233769.1673

Morales, F. (2002). El desarrollo sostenible en el Perú y la Comisión de Ambiente y Ecología. Revista del Instituto de Investigación de la Facultad de Ingeniería Geológica, Minera, Metalúrgica y Geográfica, 5(10), 80-82 https://sisbib.unmsm.edu.pe/bibvirtualdata/publicaciones/geologia/vol5_n10 /desarrollo.pdf

OCDE. (2011). Hacia el crecimiento verde. Un resumen para los diseñadores de políticas. https://www.oecd.org/greengrowth/49709364.pdf

Orihuela, C. E., \& Rojas, R. N. (2017). Desarrollo sostenible en el Perú: Evidencia del periodo 1994-2015. Anales Científicos, 78(2), 130-138. https://doi.org/10.21704/ac.v78i2.1049

Põder, T. (2006). Evaluation of Environmental Aspects Significance in ISO 14001. Environmental management, 37, 732-743. https://doi.org/10.1007/s00267- 0040190-y

Poma, Y. Y. (2018). La Gestión Ambiental Municipal y el enfoque de Ciudad Sostenible en el desarrollo ambiental de la ciudad de Huancavelica 2018. (Tesis de maestría). Universidad César Vallejo, Lima, https://hdl.handle.net/20.500.12692/34088

Raidán G (2007), Dialnet Población y Desarrollo, ISSN-e 2076-054X, ISSN 2076- 0531, No. 34, 2007, págs. 90-99 
Renjpgo, D. S., Gaviria, A., \& Baquero, O. L. (2019). Construcción de un índice de sostenibilidad ambiental y su aplicación en parcelas productivas campesinas del Municipio de Dagua. Ingeniería y competitividad, 21(2), 1- 21. https://doi.org/10.25100/iyc.v21i2i.7708

Rodríguez, J. M. (2020). Planificación ambiental. Material del curso de post grado de la maestría en Geografía, ordenamiento territorial y Medio Ambiente. Cuba: Editorial Universitaria.

Ruiz, L. (2018). La gestión ambiental en los procesos de resignificación de la existencia. Tópicos del seminario, 39, 37-64. http://www.scielo.org.mx/pdf/tods/n39/2594-0619-tods-39-37.pdf

Sánchez, H., Reyes, C., \& Mejía, K. (2018). Manual de términos en investigación científica, tecnológica y humanística. Lima, Perú: Universidad Ricardo Palma. Bussiness Support Aneth S.R.L. http://repositorio.urp.edu.pe/handle/URP/1480?show=full

Sach, I. (1980). Strategies de l'ecodeveloppement. Les Editions Ouvrieres, Paris

Stohr, W. y Todtling, F. (1979). Spatial Equality: Some Antithesis to Current Regional Development Doctrine, en Folmer, H. y Oosterhoven, J. (eds.) Spatial Inequalities and Regional Development, Nijhoff, Leiden.

Stohr, Walter B. (1981). Desarrollo desde abajo: el paradigma de desarrollo de abajo hacia arriba y de la periferia hacia adentro. Traducción ILPES. Doc. CPRD-D/80. cap. 2 de Development from Above or Below? De W.B. Storh. D.R.F. Taylor, J. Willey \& Sons Ltd.

Tinajeros, G. R. (2015). Modelo de desarrollo urbano rural sostenible para la localidad de Tarata. (Tesis de maestría). Universidad Nacional Jorge Basadre Grohmann, Tacna, Perú. http://repositorio.unjbg.edu.pe/handle/UNJBG/667

Valderrama, J. A. (2015). Conflictos entre uso actual y capacidad de uso mayor de los suelos que influyen en el desarrollo territorial sostenible del distrito de Matara, Cajamarca. (Tesis de maestría). Universidad Nacional de Cajamarca, Perú. http://repositorio.unc.edu.pe/handle/UNC/1879

Velásquez, W. (2017). Política territorial regional y su relación con la eficacia de la gestión territorial en la región San Martín - 2016. (Tesis de maestría). Universidad 
César Vallejo, Tarapoto, Perú.

ttps://hdl.handle.net/20.500.12692/31672

Vega, L. (2015). Dimensión Ambiental, Desarrollo Sostenible y Sostenibilidad Ambiental del Desarrollo. Innovation in Engineering, Technology and Education for Competitiveness and Prosperity, 1-10. http://www.laccei.org/LACCEI2013Cancun/RefereedPapers/RP256.pdf

Whittembury, K. (2021). Modelo de gestión sostenible de los residuos sólidos urbanos para el distrito de la Banda de Shilcayo, 2020. (Tesis de doctorado). Universidad César Vallejo, Tarapoto, Perú.

https://hdl.handle.net/20.500.12692/57745

Welch y Comer (1988). Coeficiente de Alpha de Cronbach. Recuperado de http://www. uv.es/ friasnav/AlfaCronbach.pdf.

Bronfenbrenner, (1987) La Teoría Ecológica de Bronfenbrenner, https://es.wikipedia.org/wiki/Teor\%C3\%ADa_Ecol\%C3\%B3gica_de_Bronfe nbrenner 